CLINICAL ALERT

\title{
Subacute sclerosing panencephalitis in South African children following the measles outbreak between 2009 and 2011
}

\begin{abstract}
E Kija, A Ndondo, G Spittal, D R Hardie, B Eley, J M Wilmshurst
Edward Kija completed his training in the Paediatric Neurology Unit, Department of Paediatrics and Child Health, Red Cross War Memorial Children's Hospital (RCWMCH) and the Faculty of Health Sciences, University of Cape Town, South Africa, where he collated the patient data included in this study. He has since returned to work as the first accredited paediatric neurologist in Dar es Salaam, Tanzania. Alvin Ndondo, a consultant in the Paediatric Neurology Unit, was integral in the clinical recognition and diagnosis of these children. Graeme Spittal, a paediatrician in the Paediatric Neurology Unit, assisted in their management. Diana Hardie works in the Division of Medical Virology in the Department of Clinical Laboratory Sciences, Faculty of Health Sciences, University of Cape Town and National Health Laboratory Service. She completed and analysed the screens undertaken to confirm the diagnosis of SSPE in these children. Brian Eley is head of the Paediatric Infectious Diseases Department in the Department of Paediatrics and Child Health, RCWMCH and Faculty of Health Sciences, University of Cape Town. He assisted in the collation of the data on the patients and relevant aspects in the literature. Jo Wilmshurst is head of paediatric neurology at RCWMCH and the Faculty of Health Sciences, University of Cape Town. She co-managed the patients, oversaw the data collation and co-ordinated the writing of the manuscript.
\end{abstract}

Corresponding author: J Wilmshurst (jo.wilmshurst@uct.ac.za)

Between 2009 and 2011, there was an outbreak of measles throughout South Africa (SA). The largest age category infected was children $<5$ years of age. In 2014, four patients, with a median age of 4 years and 5 months (range 4 years 3 months - 4.5 years), three males and one female, presented with subacute sclerosing panencephalitis (SSPE). All were infected with measles during the period of the $2009-2011$ outbreak in early infancy, at a time when their immune systems were immature and before they were vaccinated against the measles virus. One patient was immunocompromised, with vertically acquired HIV infection. All the children presented with cognitive and behavioural decline, abnormal movements and medically intractable myoclonic and atonic seizures. Outcome was poor in all and no reversibility was evident with standard therapeutic interventions. Optimal seizure control with carbamazepine is reported in patients with SSPE. Three of our patients who received carbamazepine experienced improved seizure control, but their neuroregression continued. Since submission of this case series, patient 1 (see Table 1) has died, and a further child has presented with the same clinical phenotype as described. On the basis of this clustering of patients in the Western Cape Province, SA, it is important to screen children admitted with acute cognitive decline and intractable seizures for SSPE, especially those who were infants during the measles outbreak.

S Afr Med J 2015;105(9):713-718. DOI:10.7196/SAMJnew.7788

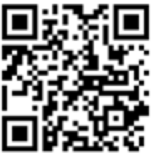

Between 2009 and 2011, 18431 laboratory-confirmed cases of measles were reported from the nine provinces of South Africa (SA) (cumulative incidence 3/100 000 population). ${ }^{[1]}$ The highest incidence was in children $<1$ year of age $(603 / 100000)$. The incidence in infants $<6$ months of age was $302 / 100000$, in those aged 6 - 8 months $1083 / 100,000$, and in those aged 9 - 11 months $724 / 100000$. Forty-eight per cent of the patients were $<5$ years of age, with the result that significant additional demand was placed on child health services. ${ }^{[1]}$ A single strain of measles virus (genotype B3) circulated throughout this outbreak.

Across SA in 2002, following the 'catch-up' campaign in 1996 - 1997, there was a $96 \%$ decrease in patients admitted with measles, and a $100 \%$ decline in reported deaths. ${ }^{[2]}$ When the outbreak in 2009 occurred, a nationwide mass vaccination campaign was therefore undertaken over a 2-week period in mid-April 2010. Concern was raised that, compared with the effectiveness of the previous vaccination campaign, the one in 2010 lacked the same uptake levels, with anecdotal reports of poor uptake on the part of some parents and schools. ${ }^{[3]}$

As an immediate consequence of the measles outbreak, a cohort of eight HIV-infected patients with probable $(n=3)$ or definite $(n=5)$ measles inclusion body encephalitis (MIBE) were managed in the neurology department at Groote Schuur Hospital, Cape Town, SA, between July and October 2010. ${ }^{[4,5]}$ MIBE manifests between 1 and 7 months after acute measles infection. Patients present with seizures, often epilepsy partialis continua, and altered cognitive awareness. ${ }^{[6]}$ The mortality rate is $85 \%$, and survivors are left with significant neurosequelae. In the case series reported from the Western Cape Province, six of the eight patients died and all followed the clinical course reported above. ${ }^{[4,5]}$ The youngest confirmed patient was 14 years of age. Anecdotally, two additional children, aged 10 (male) and 11 (female) years and suspected to have MIBE, were managed at Red Cross War Memorial Children's Hospital, Cape Town, with a parallel clinical course and findings on neuroimaging; both these patients also had HIV infection. However, serum and cerebrospinal fluid (CSF) screening in these patients, and even brain biopsy in one patient, failed to identify the measles virus. Albertyn et al..$^{[4]}$ suggested that additional cases of MIBE were probably occurring across the country, but were potentially not detected without screening for the virus or completing the required notifications; in addition, some patients may have died before reaching medical services.

Five years after the most recent (2010) measles outbreak in SA, a new cohort of children have presented with subacute sclerosing panencephalitis (SSPE). 


\section{Case summaries}

Between April and June 2014, four children were admitted to Red Cross War Memorial Children's Hospital with intractable seizures and neuroregression (Table 1). All had previously been well, and they were aged between 4 years and 3 months and 4.5 years (median 4 years and 5 months) at presentation. Typical seizures consisted of recurrent myoclonic and atonic seizures. The atonic seizures manifested as 'head nods' Cognitive function was progressively affected in all. Patient 3 had optic disc swelling at presentation, which remained static and was not associated with evidence of raised intracranial pressure. One of the children (patient 1) was immunocompromised as a result of HIV-1 infection, but had an undetectable viral load on antiretroviral therapy (ART).

CSF and serum analysis showed raised measles antibody titres, confirming the diagnosis of SSPE. Prior analysis with the polymerase chain reaction (PCR) for measles was negative on CSF in all, and initially led to the misleading assumption that the children did not have SSPE. Additional results included normal findings on CSF analysis and microscopy in all patients. CSF oligoclonal bands were positive in all the patients except patient 1 . Findings on magnetic resonance imaging (MRI) (Figs $1 \mathrm{~A}$, $1 \mathrm{~B}, 2 \mathrm{~A}$ and $2 \mathrm{~B}$ ) varied from unremarkable at presentation to the typical nonspecific white matter hyperintensities described in SSPE. ${ }^{[6]}$ Electroencephalograms (EEGs) (Figs 3 - 6B) typically demonstrated generalised periodic spike, or polyspike, wave discharges with suppression of background activity, which were intermittent and correlated with seizure events. Serial studies confirmed progressive deterioration with increasingly suppressed background activity.

Prior measles infection was rarely reported on routine history taking and had to be actively sought. Further questioning of the accompanying adults revealed that all the children had been infected with measles, either definitely or based on a history of a typical rash, between 3 months and 1 year of age. All would have received their first measles vaccination at 9 months of age, but were younger than this when they contracted measles.

Treatment in all children involved multiple combinations of antiepileptic drugs (AEDs), but with minimal response. However, improved control of myoclonic and atonic seizures was evident following the introduction of carbamazepine in three of the four children. Patient 3 remained on lamotrigine, as his seizures appeared to

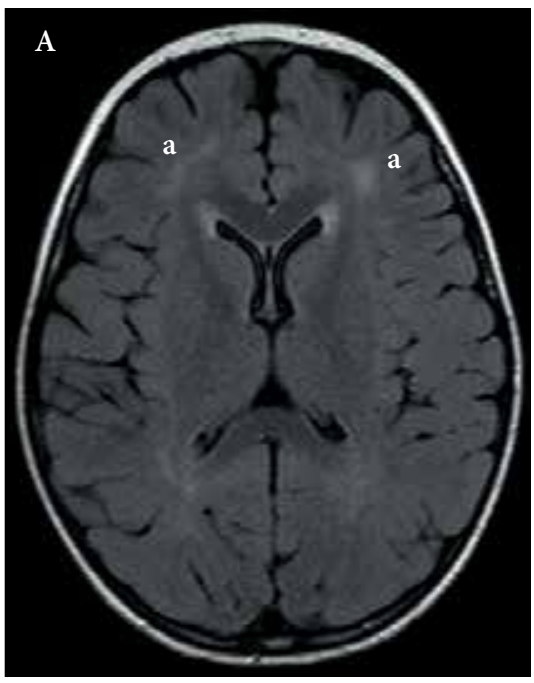

Fig. 1A. Patient 2. MRI of the brain (T2-weighted fluid attenuation inversion recovery (FLAIR)) 4 months after presentation. Imaging demonstrates nonspecific, bifrontal white matter foci of signal abnormality (a).

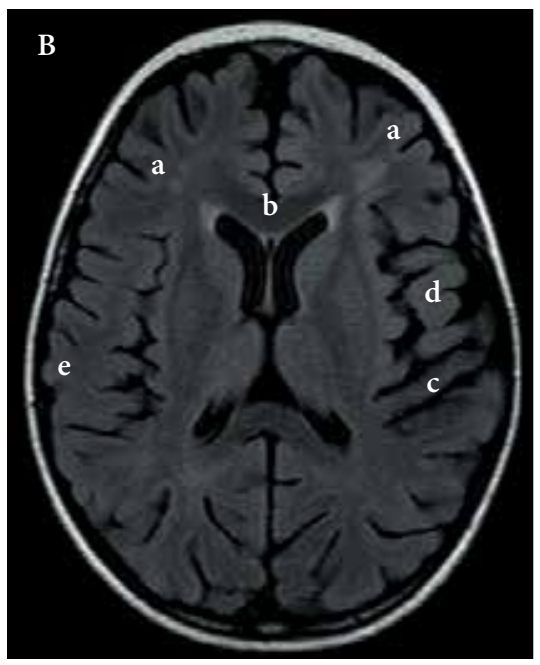

Fig. 1B. Patient 2. MRI of the brain (T2weighted FLAIR) 8 months after presentation, demonstrating an interval progression of signal abnormality in the frontal lobes (a). The imaging shows high signal, and there is corresponding restricted diffusion in the deep white matter of both frontal lobes (a), the genu of the corpus callosum (b) and the right centrum semi ovale (c). The entire right temporal lobe is now swollen with high-signal white matter and blurring of the grey/white junction (d). This area is also restricted. The medial thalami and left parietal white matter are also involved (e).

respond to this agent. In the management of patient 1 , since carbamazepine is usually avoided in combination with ART, infectious diseases specialists were consulted; given the strong data supporting the use of carbamazepine in patients with SSPE, the drug was initiated and levels of both

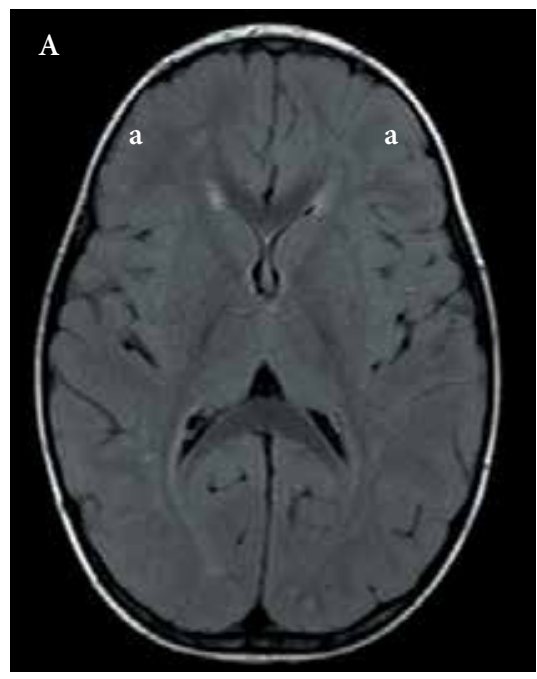

Fig. 2A. Patient 3. MRI of the brain (T2-weighted FLAIR) 1 month after presentation, showing diffuse nonspecific small areas of hyperintensity scattered in the superficial and deep white matter in the frontal temporal lobes bilaterally, with no mass effect (a).

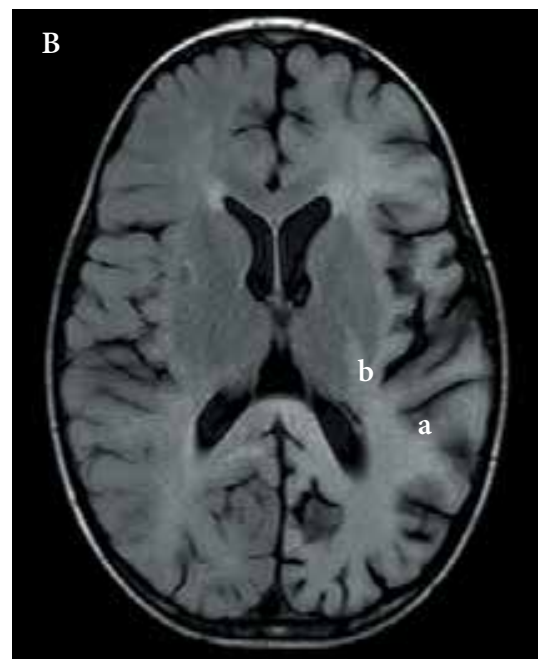

Fig. 2B. Patient 3. MRI of the brain (T2-weighted FLAIR) 6 months after presentation, showing marked interval deterioration with high signal in the white matter throughout the left cerebral hemisphere, most prominent in the parietal lobe (a). This also involves the posterior corpus callosum and posterior limb of the left internal capsule (b). Similar changes, but to a lesser extent were evident in the right parietal white matter. There was global volume loss within the left cerebral hemisphere, also involving the left cerebral peduncle and midbrain. In view of the previous measles infection, these findings were considered to be in keeping with SSPE with interval worsening.

carbamazepine and ART closely monitored. To date no further complications have occurred and the patient has experienced improved seizure control.

All the patients had progressive neurocognitive regression, loss of ambulation and speech, feeding dependency and frequent 


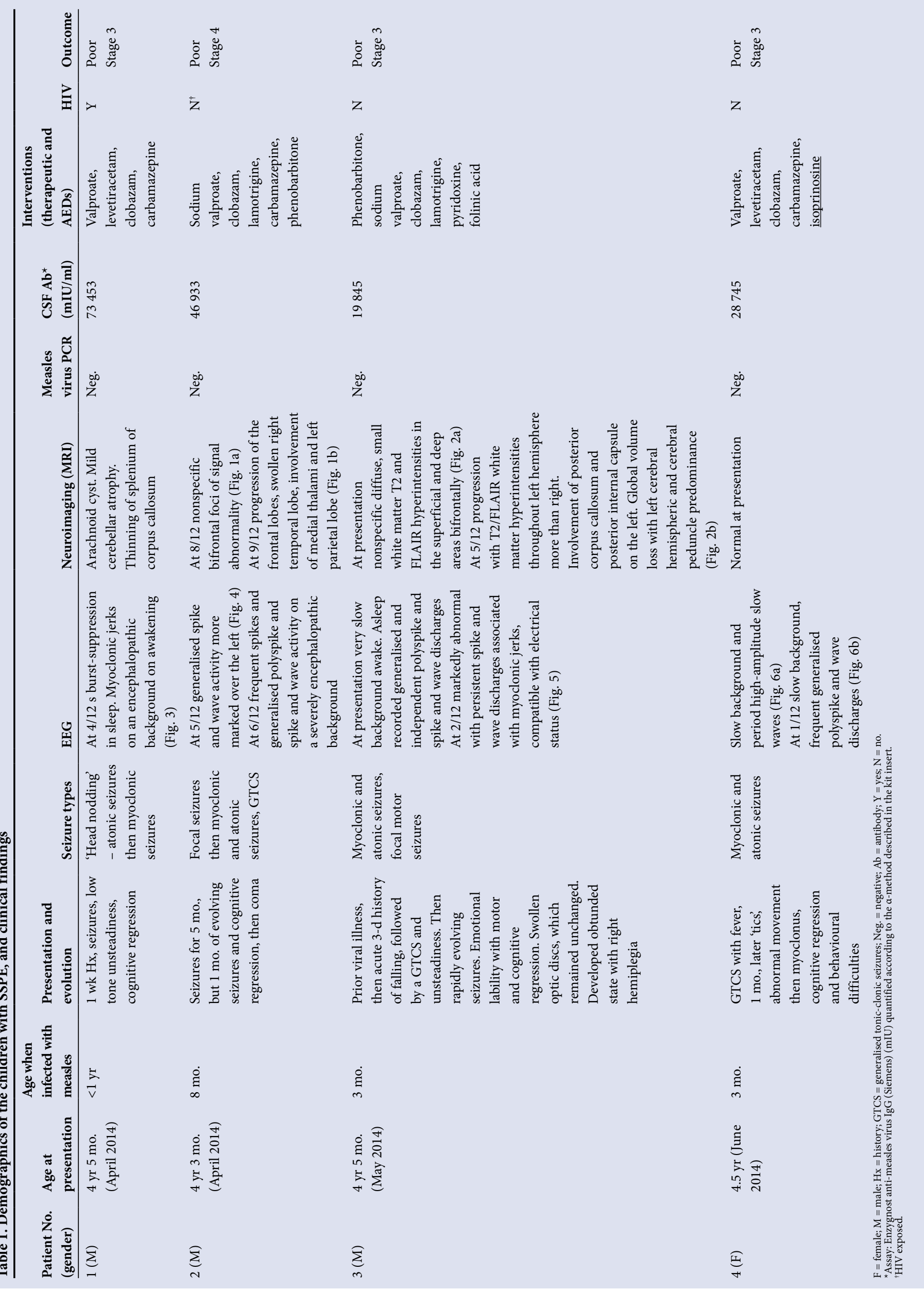




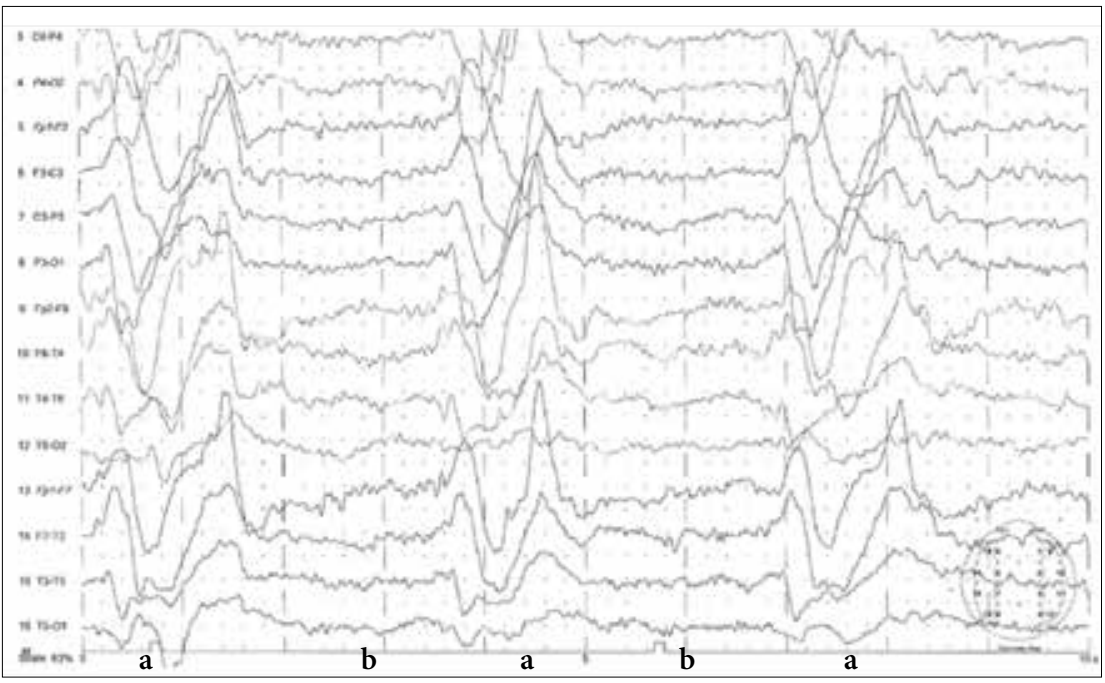

Fig. 3. EEG from patient 1 in sleep state 4 months after presentation, demonstrating profoundly attenuated background (b) with recurrent periodic generalised paroxysms (a) consistent with a burst suppression pattern.

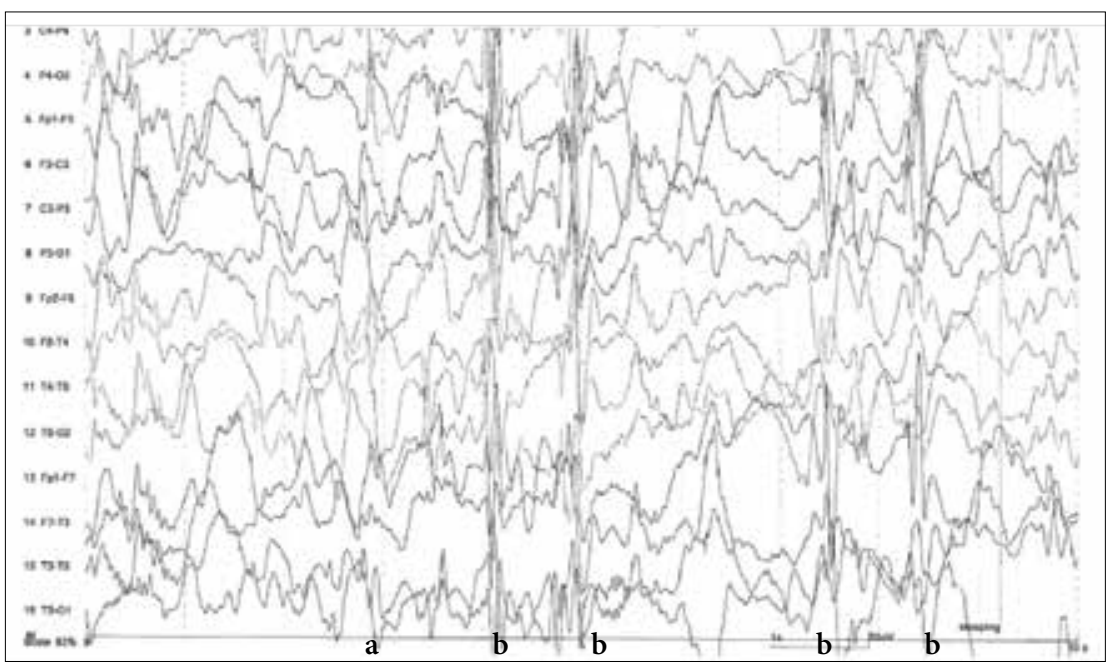

Fig. 4. EEG from patient 2 in sleep state 4 months after presentation, demonstrating slow attenuated background and recurrent periodic generalised spike and wave (a) and polyspike discharges (b) with suppression in between discharges.

seizure activity. Based on the four stages of the disease progression, as summarised in Table 2, three patients had reached stage 3 of the disease at the time of writing, and patient 2 was at the most severe stage 4 . Patient 4 was commenced on isoprinosine after the product was imported from abroad with approval from the Medicines Control Council and the local pharmacy therapeutics committee. Motivations are in place for the remaining patients.

\section{Discussion}

In children aged $<5$ years, measles is the second most common cause of death due to a vaccine-preventable disease, and it is in the top ten causes of death due to infectious diseases. ${ }^{[6]}$ The World Health Organization predicts an increase in the numbers of measles cases and deaths as a result of logistical and financial challenges affecting vaccination coverage. ${ }^{[7]}$ In 2009, the US Centers for Disease Control published a report supporting this statement, and in the same year this is exactly what transpired in SA. ${ }^{[1,7]}$ Between 2009 and 2010, 28 African countries reported measles outbreaks. ${ }^{\left[{ }^{[8}\right.}$ In Europe measles outbreaks were reported in 36 of the 53 European member states between 2009 and 2011, with the primary reason for the outbreaks reported to be failure to vaccinate susceptible populations. ${ }^{[9]}$ Vaccine safety concerns and perceived fewer benefits from vaccinations were found to be leading reasons for parents delaying vaccination of their children or refusing to vaccinate. ${ }^{[10]}$ The USA, which had declared itself 'measles eliminated' in 2000, issued a warning

\begin{tabular}{ll}
$\begin{array}{l}\text { Table 2. } \\
\text { SSPE }\end{array}$ & Clinical stages of progression of \\
\hline Stage & Clinical features \\
\hline 1 & $\begin{array}{l}\text { Mental deterioration } \\
\text { accompanied by alterations in } \\
\text { personality }\end{array}$ \\
2 & $\begin{array}{l}\text { Myoclonus and often seizures } \\
\text { Progressive neurological } \\
\text { deterioration marked by rigidity }\end{array}$ \\
4 & $\begin{array}{l}\text { Optic atrophy, akinetic mutism } \\
\text { and coma }\end{array}$
\end{tabular}

in 2014 of disease recrudescence due to imported cases. ${ }^{[11]}$ It can be concluded that this vaccine-preventable disease is far from contained.

There are three neurological complications following measles virus infection: acute disseminated encephalomyelitis, MIBE and SSPE. ${ }^{[6]}$

SSPE affects immune-competent and immune-compromised hosts. The virus is present in the brain, and the incidence is reported to be $1 / 10000$, increasing to $1 / 2500$ in children who contracted measles under 5 years of age. ${ }^{[6]}$ As in our patients, the condition occurs more commonly in boys than in girls. ${ }^{[6]}$ The risk of central nervous system (CNS) infection is increased when infection with the measles virus occurs at a young age, especially $<2$ years, when the immune system is immature and residual maternal antibodies may still be present. ${ }^{[6]}$ This was the case for the patients reported in this case series, all of whom were known or believed to have been infected with measles before 9 months of age. The pathogenesis of SSPE is poorly understood. Although viral antigen and RNA are abundant in the brain in both MIBE and SSPE, fever is unusual and the virus is difficult, if not impossible, to culture from CNS tissue. Resource-poor settings tend to have a higher incidence of measles infection than high-income countries and carry a higher burden of SSPE, although the condition may not be readily diagnosed. ${ }^{[6]}$ It is not unusual for the diagnosis of SSPE to be challenging and delayed owing to preferential consideration of other diverse differential diagnoses. Over $78 \%$ of patients were misdiagnosed in one large study of 307 patients, with the median time to diagnosis of SSPE being 6 months (range 0.2 - 96). ${ }^{[6]}$ Similar challenges were evident with our patients. Even when the diagnosis was suspected, there were a number of barriers to diagnostic closure. Furthermore, in settings such as SA where there is a high burden of HIV infection, the 


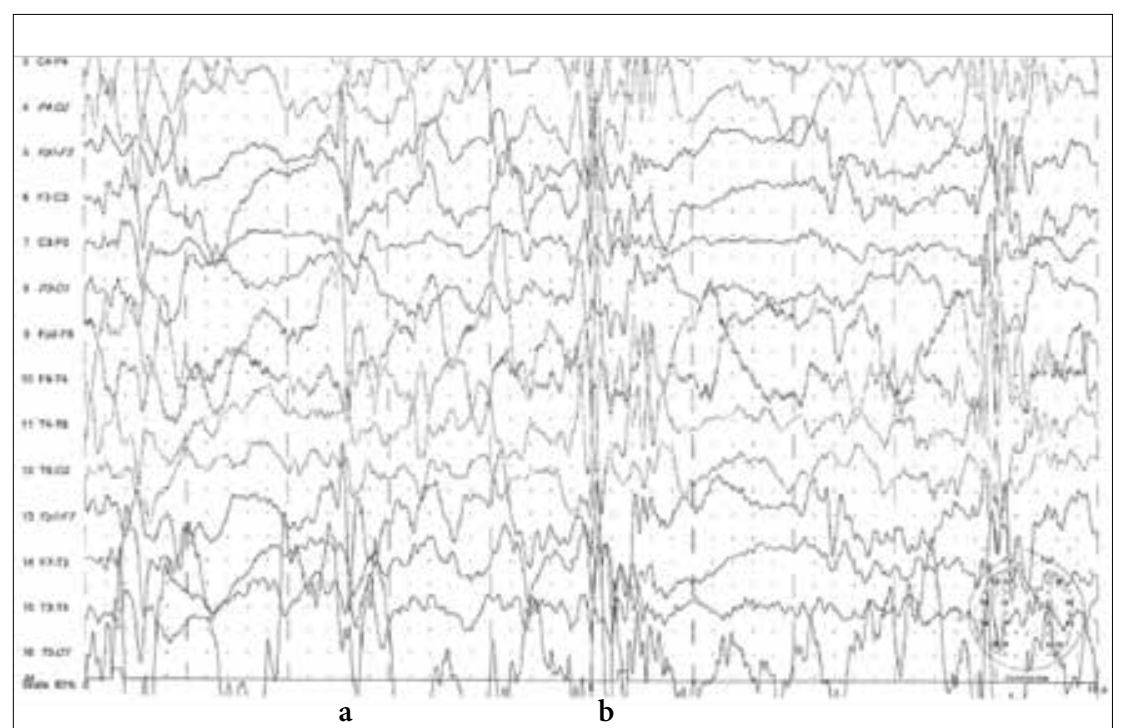

Fig. 5. EEG of patient 3 in sleep state 2 months after presentation, showing a markedly abnormal recording with persistent spike and wave discharges (a) associated with myoclonic jerks and suppression (b) and compatible with electrical status.

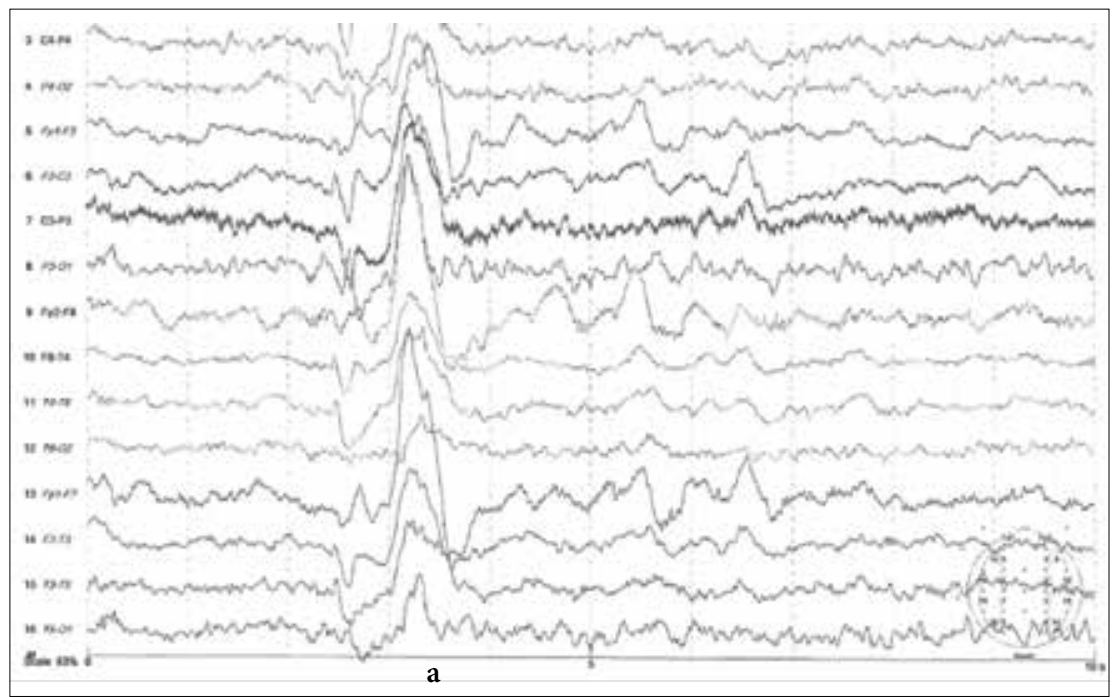

Fig. 6A. Patient 4. EEG at presentation in sleep state, demonstrating a slow attenuated background with a generalised high-amplitude slow wave (a) which occurred periodically throughout the recording.

incidence may be expected to be further increased. Children of HIV-infected mothers are at particular risk of acquiring measles early, even before 9 months of age, which is the recommended age for vaccination in resource-limited settings. ${ }^{[6]}$

The average time to onset of SSPE after measles is 6 - 10 years (range 1 - 24). ${ }^{[6]}$ The onset is insidious and the diagnosis is often not suspected early in the disease. ${ }^{[6]}$ All the children in our series presented before 6 years of age and the SSPE followed an aggressive course, rapidly reaching either stage 3 or 4 disease. Patient 3 presented with swelling of the optic discs, which was identified during his acute management when he presented with seizures and regression. This finding, in combination with positive oligoclonal helpful and supportive of the diagnosis of SSPE. Neuroimaging is generally unhelpful, findings often being normal in the early stages of the disease, and the focal or diffuse T2-weighted hyperintense lesions that appear later are not pathognomonic. ${ }^{[6]}$ This was the case in our patients; even patient 3, who developed extensive white matter hyperintensities, would have had a wide range of differential diagnoses without the finding of raised CSF measles antibodies that supported a diagnosis of SSPE. While in our patients the seizure presentation was a major indicator of a more complex condition, all had been well before their abrupt onset of cognitive dysfunction and seizures. Their seizures were very stereotyped, persistent and resistant to typical medical interventions. Despite relatively early suspicion of SSPE, the initial lack of history of infection and negative CSF PCR results were misleading. It was only with persistence (largely on the part of author AN) that the history of prior measles infection was confirmed in all, after which pursuit of the correct screening in the CSF, namely the measles antibody titres, enabled the diagnosis to be confirmed. Viral nucleic acid is rarely found in the CSF of patients with SSPE. ${ }^{[14]}$ This is due to absence of a typical productive infection of the brain with extracellular release of virus. In the case of patients with SSPE, only the genome and nucleocapsid replicates and these spread from cell to adjacent cell through membrane fusion across synapses. Viral nucleic acid will therefore only be present in infected cells. Findings were similar in the patients reported with MIBE, of whom only two were PCR-positive in the CSF. ${ }^{[5]}$

Numerous therapeutic agents, including amantadine, interferon, isoprinosine and ribavirin, have been used for treatment of SSPE. Evaluation of efficacy is difficult, as most cases are isolated and occur in small clusters. ${ }^{[6]}$ The most commonly used regimen is a combination of isoprinosine and interferon-alpha, with some suggestion that this combination slows disease progression. ${ }^{[6]}$ The latter intervention is not available in SA.

Turkey is in the unfortunate position of having significant experience in the condition. Guler et al ${ }^{[15]}$ reported 64 patients with SSPE, diagnosed at an average age of 12.3 years (range 5 -17). None of the interventions attempted, including isoprinosine and interferon, altered the longterm outcome. Furthermore, there was no difference between those who received isoprinosine in isolation and those receiving isoprinosine and interferon in combination. Guler et al. commented on high levels of 


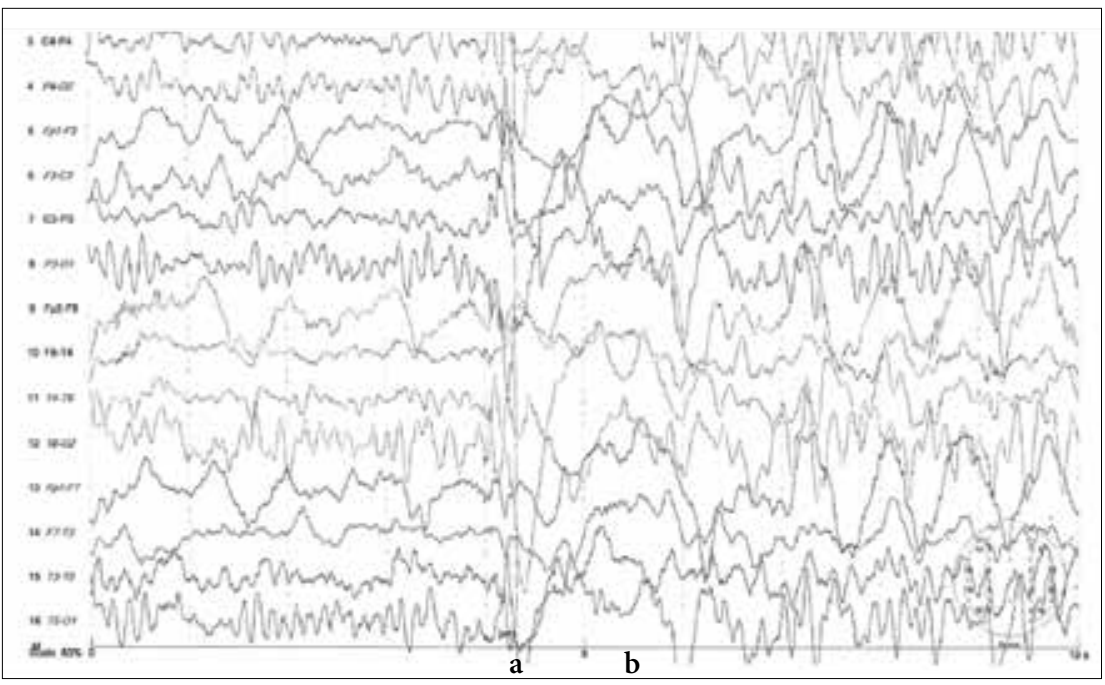

Fig. 6B. Patient 4. Follow-up EEG at 1 month in awake state, demonstrating normal posterior alpha rhythms with overriding $2 \mathrm{~Hz}$ generalised slow waves and periodic spike and slow wave (a) followed by 2 seconds of attenuation (b). This discharge correlated with a myoclonic seizure and was recurrent throughout the recording.

consanguinity, and questioned whether an additional genetic modifying factor in their patients made them more susceptible to developing SSPE. A study from Karachi in Pakistan reported on 43 patients with similar clinical findings and outcomes. ${ }^{[16]}$

There are several implications for management, which is significantly determined by confirmation of the diagnosis, beyond the importance to the family of having diagnostic closure. Presentation with myoclonic or atonic seizures would normally preclude the use of carbamazepine, but for patients with SSPE this agent has the best seizure efficacy. ${ }^{[17-20]}$ For our patient with HIV this led to further complications relating to the need for more aggressive monitoring of his HIV viral titres and the carbamazepine levels, owing to the risk of cross-reactivity with ART. An alternative agent could have been levetiracetam, but in fact careful monitoring of drug levels enabled carbamazepine to be used successfully. There are no data in the literature to guide a treatment care plan in the setting of SSPE and the immunocompromised patient, and colleagues in Turkey had not managed such a case (personal communication, B Anlar). This scenario is likely to be a uniquely African challenge.
Routine measles vaccination is the best approach for preventing SSPE. ${ }^{[6]}$ Measles vaccine is a live attenuated vaccine, and vaccine strains have not caused SSPE. Furthermore, it is widely accepted that SSPE cannot occur in the absence of direct infection with wild-type measles virus. ${ }^{[6]}$ The incidence of SSPE is directly related to the incidence of measles in a population, and has decreased dramatically since the introduction of measles vaccination. ${ }^{[6]}$

The four cases described here may represent only a proportion of the cases across SA. In addition, it is likely that the reported numbers of children infected during the measles outbreak are an underestimation, and further patients are therefore likely to present with SSPE. Children presenting with acute-onset intractable seizures, especially myoclonus and atonic siezures, and showing neuroregression should have a careful history of prior measles infection in infancy documented, and there should be a low threshold for screening for measles CSF antibodies.

\footnotetext{
1. Ntshoe GM, McAnerney JM, Archer BN, et al. Measles outbreak in South Africa: Epidemiology of laboratory-confirmed measles cases and assessment of intervention, 2009-2011. PLoS One 2013;8(2):e55682. [http://dx.doi.org/10.1371/journal. pone.0055682]
}

2. Uzicanin A, Eggers R, Webb E, et al. Impact of the 1996-1997 supplementary measles vaccination campaigns in South Africa. Int J Epidemiol 2002;31(5):968-976. [http://dx.doi.org/10.1093/ ije/31.5.968]

3. Siegfried N, Wiysonge CS, Pienaar D. Too little, too late: Measles epidemic in South Africa. Lancet 2010;376(9736):160. [http:// dx.doi.org/10.1016/S0140-6736(10)61100-2]

4. Albertyn C, van der Plas H, Hardie D, et al. Silent casualties . Albertyn C, van der Plas $\mathrm{H}$, Hardie D, et al. Silent casualties
from the measles outbreak in South Africa. S Afr Med J from the measles outbreak in

5. Hardie DR, Albertyn C, Heckmann JM, Smuts HE. Molecular characterisation of virus in the brains of patients with measle inclusion body encephalitis (MIBE). Virol J 2013;10:283. [http:// dx.doi.org/10.1186/1743-422X-10-283]

6. Griffin DE. Measles virus and the nervous system. Handb Clin Neurol 2014;123:577-590. [http://dx.doi.org/10.1016/B978-0444-53488-0.00027-4]

7. Centers for Disease Control and Prevention (CDC). Global measles mortality, 2000-2008. MMWR Morb Mortal Wkly Rep 2009;58(47):1321-1326. http://www.cdc.gov/mmwr/preview/ mmwrhtml/mm5847a2.htm (accessed 6 August 2015).

8. Centers for Disease Control and Prevention (CDC). Measles outbreaks and progress toward measles preelimination African region, 2009-2010. MMWR Morb Mortal Wkly Rep African region, 2009-2010. MMWR Morb Mortal Wkly Rep
2011;60(12):374-378. http://www.cdc.gov/mmwr/preview/ 2011;60(12):374-378. http://www.cdc.gov/mmwr/
mmwrhtml/mm6012a3.htm (accessed 6 August 2015).

9. Centers for Disease Control and Prevention (CDC). Increased transmission and outbreaks of measles - European Region, 2011. MMWR Morb Mortal Wkly Rep 2011;60(47):1605-1610. http://www.cdc.gov/mmwr/preview/mmwrhtml/mm6047al. htm (accessed 10 August 2015).

10. Smith PJ, Humiston SG, Marcuse EK, et al. Parental delay or refusal of vaccine doses, childhood vaccination coverage at Rep 2011:126(Suppl 2):135-146. http://www.ncbinlm.nih gov/ pmc/articles/PMC3113438/pdf/phr126s20135.pdf (accessed 6 pmc/articles/P

11. Thaku K. World Neurology: Re-emergence of measles in the United States: A warning to neurologists. 2014. http://www United States: A warning to neurologists. 2014. http://www.
worldneurologyonline.com/article/re-emergence-measles-unitedworldneurologyonline.com/article/re-emergence-measles- $\mathrm{t}$
states-warning-neurologists/ (accessed 20 November 2014).

12. Yuksel D, Sonmez PA, Yilmaz D, Senbil N, Gurer Y. Ocular findings in subacute sclerosing panencephalitis. Ocul Immunol Inflamm 2011;19(2):135-138. [http://dx.doi.org/10.3109/092739 48.2010.535636]

13. Oray M, Tuncer S, Kir N, Karacorlu M, Tugal-Tutkun I. Optic neuritis and rapidly progressive necrotizing retinitis as the initial signs of subacute sclerosing panencephalitis: A case report with clinical and histopathologic findings. Int Ophthalmo 2014;34(4):983-987. [http://dx.doi.org/10.1007/s10792-014-9914-7]

14. Yanagi $\mathrm{Y}$ Takeda $\mathrm{M}$, Ohno $S$, Hashiguchi $T$ Measles virus receptors. Curr Top Microbiol Immunol 2009;329:13-30. [http:// dx.doi.org/10.1007/978-3-540-70523-9_2]

15. Guler S, Kucukkoc M, Iscan A. Prognosis and demographic characteristics of SSPE patients in Istanbul, Turkey. Brain Dev characteristics of SSPE patients in Istanbul, Turkey. Brain Dev
201537(6):612-617. [http://dx.doi.org/10.1016/.braindev.2014.09. 201537(6):612-61,
006] Sep 27,

16. Rafique A, Amjad N, Chand P, et al. Subacute sclerosing panencephalitis: Clinical and demographic characteristics. J Coll Physicians Surg Pak 2014;24(8):557-560. [08.2014/ JCPSP.557560]

17. Hayashi $T$, Ichiyama $T$, Nishikawa $M$, Furukawa $S$. Carbamazepine and myoclonus in SSPE subacute sclerosing panencephalitis. Pediatr Neurol 1996;14(4):346. [http://dx.doi. org/10.1016/0887-8994(96)00086-0]

18. Yigit A, Sarikaya S. Myoclonus relieved by carbamazepine in subacute sclerosing panencephalitis. Epileptic Disord 2006;8(1):77-80. http wwwjle.com/en/rovues/epd/e-docs/myoclos www.jle.com/en/revues/epd/e-docs/myoclonus_relieved_by carbamazepine_in_subacute_sclerosing_panencephalitis_268140/
article.phtml (accessed 6 August 2015). article.phtml (accessed 6 August 2015).

Aydin OF, Senbil N, Gurer YK. Nonconvulsive status epilepticus on electroencephalography in a case with subacute sclerosing
panencephalitis. J Child Neurol 2006;21(3):256-260. [http:// panencephalitis. J Child Neurol 2006,
dx.doi.org/10.2310/7010.2006.00056]

20. Ravikumar S, Crawford JR. Role of carbamazepine in the symptomatic treatment of subacute sclerosing panencephalitis A case report and review of the literature. Case Rep Neurol Me 2013;2013:327647. [http://dx.doi.org/10.1155/2013/327647]

Accepted 18 December 2014 